\title{
Using Social Metadata in Email Triage: Lessons from the Field
}

\author{
Danyel Fisher ${ }^{1}$, A.J. Brush ${ }^{1}$, Bernie Hogan ${ }^{2}$, Marc Smith ${ }^{1}$, and Andy Jacobs ${ }^{1}$ \\ ${ }^{1}$ Microsoft Research, One Microsoft Way, Redmond, Washington. USA \\ ${ }^{2}$ Department of Sociology, University of Toronto, 725 Spadina Avenue \\ Toronto, ON M5S 2J4, Canada \\ \{danyelf, ajbrush, masmith, andyj\}@microsoft.com, \\ bernie.hogan@utoronto.ca
}

\begin{abstract}
Email archives are full of social information, including how messages are addressed and frequency of contact between senders and receivers. To study the use of this rich metadata for email management, particularly email triage, we deployed SNARF, a prototype tool which uses social metadata to organize received email by correspondent, sort received email by past interactions, and filter email into multiple views. We discuss the lessons from a seven month deployment, including the value of organizing by personally addressed mail, the unexpected value of SNARF for email awareness, challenges with handling workflow, and ways to use social metadata in applications.
\end{abstract}

Keywords: Email overflow, email triage, social metadata, social sorting.

\section{Introduction}

The second time we meet a friend's dog it typically remembers us and so does not growl. Remembering how we have interacted with others in the past is basic to social interaction. Much like in-person interaction, email communication is rich with social information. How frequently have you previously corresponded with a particular sender? Was the message addressed directly to you or to a mailing list? Are you the only recipient? Unfortunately, email clients typically ignore this social metadata the information about both a persons' history of interaction with their correspondents, and the ways that a message is addressed - treating all messages equally without regard to whether they come from a frequent correspondent or new friend.

During the task of email triage, people seek to separate relevant messages from a swath of less relevant messages. Neustaedter et al. [10] suggested that many social aspects of an email message are important to users when deciding what email to read during email triage including the sender and how the email was addressed to the recipient. That research, as well as others [15], also found that many users choose to employ a multi-pass strategy to read their email, skimming through to select ones that are particularly easy to handle or particularly important. Additional passes-to choose 
the next most important message-follow as time permits. Unfortunately, most email clients have limited support for the multi-pass practice, presenting email in order of receipt, rather than relevance.

While several research systems [7, 13] use social metadata in various ways, contemporary email clients do not provide users a way to directly view or access social metadata, nor do they utilize interaction histories. (This information is sometimes indirectly used to help fight spam.) Indeed, current email tools provide few social cues: besides instant messaging icons, email tools rarely give any feedback about our correspondents at all.

Although email facilitates communication and collaboration, email management is an intensely personal practice. To study the value of social metadata for email triage in a real world environment over time we extended and deployed a prototype application, SNARF, the Social Network and Relationship Finder [11]. At the core, SNARF implements a simple idea: present email organized by sender in lists that are ordered by the strength of relationship between the sender and user. Email from a colleague one corresponds with frequently will appear higher in the list than email from an infrequent correspondent. SNARF, then, uses the frequency of communication as an (imperfect) proxy for relationship strength.

SNARF employs social metadata to present users with alternative views of their email in three ways. It filters email by whether it has been read and how it is addressed; it organizes email by sender; and it sorts senders based on their history of interaction with the user. By allowing the user to focus on contextually-appropriate messages, and by bringing messages from frequent correspondents to the fore, we believe that SNARF allows users to more easily locate relevant messages, especially when pressed for time. SNARF supports the advantages of a multi-pass strategy without requiring users to repeatedly scan their entire inbox.

Over the course of seven months and two iterations of SNARF we gathered feedback through surveys, usage logs, and user suggestions from nearly a thousand people in our organization about their experience with SNARF.

The feedback (both positive and negative) that we collected from people using SNARF on their own email in everyday life taught us several lessons about the value of social metadata for email management. Most popular among SNARF users were the way to filter for messages explicitly addressed to themselves. Somewhat surprisingly, several SNARF users made unanticipated use of the tool to help with awareness of new mail. We also saw challenges for making use of social metadata around identity, particularly when people use multiple email addresses, and when people dramatically change their communication context, as when taking a new job.

In the rest of the paper we discuss related work (Section 2) and then describe the design of SNARF (Section 3) and our field deployment (Section 4). Section 5 presents our key findings from the field study and highlights how our experience with social metadata can help other designers and developers make informed decisions on how and when to incorporate social metadata in their applications (Section 6). 


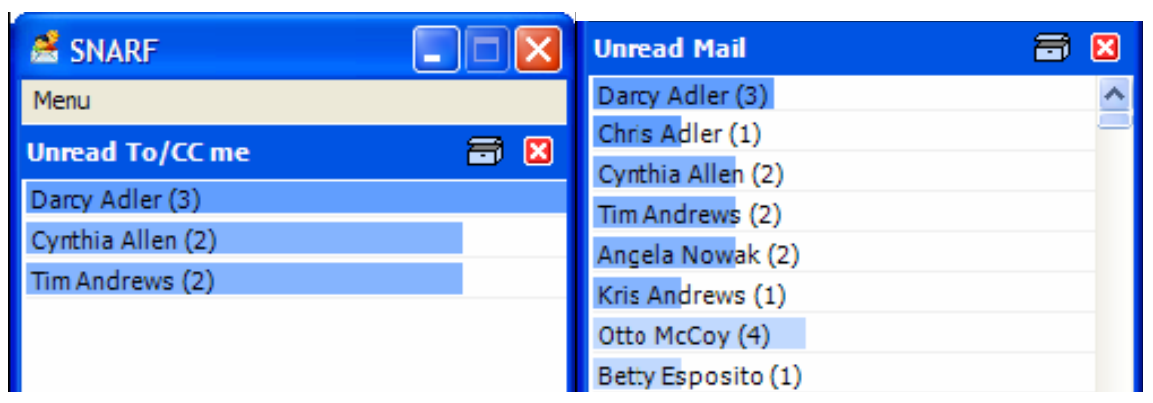

Fig. 1. Two views in the SNARF Interface: names are presented along with unread counts. Ordinarily, these two boxes would be arranged in a narrow vertical column.

\section{Related Work}

Assisting users in working with email has been addressed in many different ways in the HCI field from innovative interfaces [2, 7] to prioritizing systems [8]. IBM's ReMail [7] prototype includes a Correspondent Map, which organizes email by correspondent and organizational affiliation. Tyler and Tang [13] evaluates email histories to predict future availability and responsiveness. Nardi et al. [9] suggest that contact management takes a substantial amount of effort, and propose a sociallybased interface that shows information oriented around people. In SNARF, we adapt this notion of orienting an interface around correspondents (rather than messages) for handling email.

\section{Design of the SNARF Prototype}

Takkinen and Shahmehri [12], followed by Venolia et al. [15] discuss multiple phases of handling email. SNARF is designed for email triage (or "busy mode"), a time when the user needs to locate particularly important messages and handle them quickly, and is to be used in addition to (rather than instead of) traditional email programs. The technical basis of SNARF's design was discussed in [11]. Here, we focus on the ways that SNARF uses social metadata to present email and the features needed to support day to day use in the field deployment.

\subsection{Use of Social Metadata Within SNARF}

SNARF uses information about past email behavior to display a user's email in three main ways.

Filtering Mail into Views. The main SNARF window is divided into several panes, each presenting one view showing a subset of available email correspondents ${ }^{1}$. Views filter mail based on how it was addressed: they separate mail that is addressed directly

\footnotetext{
${ }^{1}$ An email address can refer to a person, a mailing list, or an automated generator. For
} simplicity, we refer to all senders and receivers as correspondents. 
to the user-mail that is more likely to invoke a new task-from mail that may not be as critical. In general, views are based on a collection of sorting and filtering settings.

Figure 1 shows the default configuration of SNARF, with three views. The top view, "Unread To/CC me," shows correspondents whose unread messages have explicitly included the user in the To or CC line. The middle view, "Unread Mail," shows unread messages from all correspondents (and thus may have been received through a mailing list). The bottom view, "This Week's Mail," shows all correspondents from whom the user has received messages in the last week, read or unread. The panes are not mutually exclusive; a particular message or person may appear in multiple panes if they match the criteria.

The original design for SNARF [11] showed only one view at a time; users would re-configure the display to match their current task. However, as the design evolved it became apparent that it is important to have multiple views so that more types of sorts could be shown. This reflects the difference between the relevance of a particular sender, and a sender's message. For example, Chris Adler may be very important to me, but messages from Chris sent only to me are often qualitatively different from messages sent by Chris to a mailing list we both subscribe to. The current display, which can accommodate three views of eight or so names without scrolling on a conventional screen, reflects this design.

Organizing by Correspondent. Messages are clustered together and linked to the sender of the message, in order to present a social perspective on the email. Each correspondent name is followed by the number of unread messages from them.

Sorting by Correspondence History. Each view presents names socially sorted: ordered by the degree of relation to the user that can be extracted from their interaction history. In the default view, names are ordered by the number of times that the user has sent mail to that person in the past year, although the time period is configurable. Frequent and recent correspondents bubble to the top, while infrequent correspondents sink to the bottom. In Figure 1, in the To/CC me view, the user has sent more email messages to Darcy Adler than to Tim Andrews; Darcy is therefore sorted higher on the display than Tim. The configuration options available on this display are discussed in more detail in [11].

Design for Field Deployment. In order to deploy SNARF, the prototype needed to be a tool that could support real day-to-day use. In particular, this required SNARF to respond to real-time updates and the ability to open mail and read it. SNARF therefore is a MAPI client, and connects itself automatically to the users' Outlook installation. The several views, like all aspects of SNARF, respond in real time to changes in email state. For example, if a message from a sender to the user is marked as read, the "Unread Messages" view is updated-it will no longer show that sender if no more unread messages remain.

When the user double-clicks on an entry showing just one message, SNARF opens the message in Outlook. If the correspondent is associated with more than one message - that is, there is more than one unread message from that person-then SNARF presents a message list in a second window. 


\section{Field Deployment}

We gathered data about SNARF's use in the field in two phases. The first phase was a structured field study that took place over a six week period in July and August of 2005 where we conducted pre-surveys and post-surveys of SNARF users. Once the structured field study concluded, we shifted to a second phase where we released an updated version of SNARF and collected usage logs.

Phase 1: Field Study. The field study began in July 2005 when we sent out an invitation to use SNARF to people who had registered their interest at an internal demonstration of the system, as well as to two high-volume mailing lists of employees at our company that specifically target people with interest in advanced and speculative projects. In total, 574 people ran SNARF at least once within the first two weeks after it was made available. We stopped accepting new people into the study after two weeks, although they could still use SNARF. During the study we explicitly chose to make only one change to SNARF to address a performance issue when opening the message list window.

Data Collection. All users that ran SNARF in the first two weeks of the study were asked to complete an optional pre-survey related to their experiences with email. The survey followed the outlines of [10]; however, it added more detailed information about email habits within the user's current client. The survey included questions asking users how long they thought they spent triaging email, how much of their email they read, and their experience of stress associated with handling email.

In August 2005, at the end of the six-week study period, we sent out a post-survey. Users were asked to respond regardless of whether they had filled out the pre-survey or had continued to use SNARF after its initial installation. All users who reported that they used SNARF 'for a few days' or more were asked about their use in more detail on the post-survey. Users were asked to respond regardless of whether they had filled out the pre-survey or had continued to use SNARF after its initial installation. Participants that filled out the post-survey were entered into a lottery for gift certificates.

Study Experience. During the study we received extensive feedback from SNARF users. A total of 292 people completed the pre-survey, a response rate of $51 \%$ and 161 people filled out the post-survey (response rate of $28 \%$ ), some of whom had not responded to the pre-survey. Post-survey respondents identified their job role as largely program managers (23\%), developers (17\%) and consultants (13\%); sales $(10 \%)$ and software testers $(9 \%)$ made up the bulk of the remainder. The population was overwhelmingly male $(92 \%)$.

Post-survey responses help us understand SNARF adoption. Of the respondents $4 \%$ of users reported that they could not get SNARF to work, $44 \%$ 'tried it once or twice', $29 \%$ tried it 'for a few days', $11 \%$ tried it 'for a few weeks' and $13 \%$ were 'still using SNARF'.

Phase 2: Ongoing SNARF Use. After the field study concluded, we released a series of updates to SNARF which addressed issues that the first version revealed. We promoted and released an improved SNARF several months later. 410 users tried the program at least once and provided log data during this second phase, which ran from 
late November to February of 2006. Usage data was logged and uploaded daily containing records of both any changes to the mail store and click data within the program itself. Of the 410 users, $25 \%$ used it on five or more days (the maximum was 103 days, almost seven days a week). The usage data provides a complementary picture to the qualitative survey data.

\section{Lessons from the Field}

In this section we describe the lessons we have learned from our seven month field deployment of SNARF, bringing together qualitative data gathered in the field study phrase and quantitative data from user logs as well as feedback provided directly to us by users. We classify our observations into a number of different aspects of using social history, including social filtering, social awareness, and email workflow. We examine places where SNARF has succeeded-and ways in which it has not.

\subsection{Social Filtering}

SNARF uses social filtering to give users multiple views of their email. We learned that people are particularly focused on whether a message is addressed to them and they took advantage of having multiple views. We also saw that the interface must be careful to support multiple overlapping views in a way that is understandable to the user.

Personal Mail Matters: “[My favorite aspect of SNARF was:] Showing the email that was just to me or cc to me." SNARF provides many different configurable views. Conceptually, we separate these views into two types. Personal views filter for messages that explicitly mention the user, such as "Unread To/CC me." In contrast, aggregate views show collections of messages are not addressed to the user, such as unread messages sent to lists ("Unread lists"). Personal views generally contain shorter lists of people; the latter type generally contains more people. On both the post survey and in the usage logs, we saw a preference for personal views

Multiple Views Were Used. SNARF provides multiple views of a person's email. On the survey, users "agreed" (mean $=1.17, \mathrm{~N}=69^{2}$ ) that "it is useful to filter mail into a number of panes." Of the 358 people that clicked once in any view, 225 of them clicked at least once in two or more views. This supports the idea that maintaining multiple perspectives on email is valuable.

Users found different views useful for different tasks. During the triage task, for example, users might concentrate on mail sent directly to them as they cleared out messages that directly needed their attention. List views were particularly useful for catching up on a high volume of mailing list messages. Skimming the top off the "Unread Mail" view allows a user to monitor interactions on mailing lists and discussion that involve their closet correspondents.

${ }^{2}$ On a -2 ("Strongly disagree") to +2 ("strongly agree") scale. This scale is used for all survey responses unless noted. The words "agree," "strongly agree", and similar in quotation marks refer to the median choice. 
Challenges Showing Messages Multiple Places. Some users complained that they would see the same message in several different places, as the "Unread mail" would show messages that we also "To/CC Me". The experience of reading one message and having several parts of the interface change can be distracting or confusing. While SNARF does not fully handle this situation, any system that repeats information in multiple places must make this clear.

\subsection{Organizing by Correspondent}

SNARF supports the idea of organizing information socially. [10] suggested that users pressed for time feel a need to "cherry-pick" their mail, seeking out messages specifically from people they know are relevant. SNARF cherry-picks for them, first separating messages that are directly to them, then ordering sets of messages by how often the user and sender correspond with one another.

While we feared that users might complain that clustering by person would roll together irrelevant ("Lunch!") messages with relevant ("Need report soon"), users "agreed" (mean $=1.08, \mathrm{~N}=73$ ) that listing "correspondents rather than individual messages is useful."

\subsection{Social Sorting}

SNARF shows that the world is divided into those people who I have previously interacted with and those who I have not. People who I have not sent mail to before are treated differently than people who I have. This is the concept behind white-lists, which are meant to hold off spam and bulk email; it can also be successful in handling email messages during triage. During those pressed times, emphasizing the top few messages from the most-contacted correspondents probably makes sense.

The goal of social ranking in SNARF is that the high-priority people a user interacts with often will float to the top of a list, while strangers would stay at the bottom. Survey respondents generally "agree" (mean $=0.95, \mathrm{~N}=58$ ) that sorting correspondents socially is useful. One user commented, "[My favorite aspect of SNARF was:] sorting mail by sender, first sender first."

Organizational Information and Sorting. The default sorting in a SNARF view is based on the number of messages a user has sent to a correspondent. This method of sorting neglects to take into account important hierarchical information about organizational relationships. We have chosen not to incorporate organizational information into SNARF at this point. In practice, we have found that multiple views may handle issues of important email from an infrequent correspondent. As personal views are typically shorter (section 5.1.1), a personally addressed message from an important but infrequent correspondent is easily spotted.

\subsection{Social Awareness: An Unexpected Gain}

Perhaps the most surprising result of the deployment of the SNARF tool was the success of using SNARF as an awareness tool. The same minimalist display that addressed the triage task also meant that the application could be left active at a minimal cost of screen space. Post-survey respondents that told us they were "still 
using" SNARF at the time of the survey "agreed" that the tool was useful for keeping aware (mean $=0.78, \mathrm{~N}=18$ ). In contrast, users who used SNARF just for "a few days" before stopping "disagreed" that SNARF was useful for keeping aware (mean = -0.43 , $\mathrm{N}=40$ ), a lower rating than they gave to "finding important email" or "triaging."

SNARF could have been helping handle the "flow" phase of email (to use [12] terminology): the time in which users keep up with incoming messages, is also a poorly-addressed feature in many current tools. For example, in many common mail tools, a small notification window appears in the corner of the screen for a short time when new mail arrives, and disappears shortly after. This is a "push" mechanism: if the user is to read the message, they must quickly evaluate the window, reading the sender and subject, decide if they wish to read it, and click, all within a few seconds. In contrast, SNARF shifts to a "pull" model. A user can glance at SNARF to see if new email they might be interested in has arrived and then decide whether to read the message now or later, without the sound or a distracting [3] visible change.

\subsection{Email Management and Workflow}

The email triage task, as explored by $[10,12,15]$, focuses on deciding how to handle individual messages. The notion of a mailbox needing to be cleaned up or reorganized is left for a periodic "cleanup" sweep. In reality, users are accustomed to often being able to file or delete messages after they have been read, even if they often do not bother to do so.

Many "one-touch" strategies for dealing with email (such as the popular "Getting Things Done" [1]) combine reading mail with immediate filing or processing of messages. Many users seemed accustomed to reading a message, closing it, and then filing or deleting it. Once the message was read in SNARF, it was no longer able to be deleted, as it had disappeared from the interface. Users who wanted to delete messages would have to go to Outlook to do so. This meant users found that SNARF did not smoothly support the filing and processing phase of triage.

In addition, the inbox being a "habitat" [4] means that already read messages can be important for browsing or reviewing for tasks. Once a message had been read, it disappeared from the SNARF interface, making it poor for reviewing current tasks.

\subsection{Social Metadata Challenges}

Changes in Context. As we note above, social histories by necessity track a user's past. Yet sometimes, a user's context changes - by changing jobs, for example, or by switching projects - and their history can become less valuable as predictors of the relevance of content from new correspondents. Socially-sorted systems should consider ways to resolve this, either by reducing the importance of past interactions, by pre-populating information, or by incorporating organizational information.

Social History is More Than Email. SNARF treats the volume of past emails as a proxy for relationship strength. Obviously, this is a fairly limited metricrelationships and interactions with other people are far deeper, as anyone with a parent who dislikes email knows. SNARF's logs do not capture instant message conversations, telephone calls, or hallway interactions. It is clear that measuring 


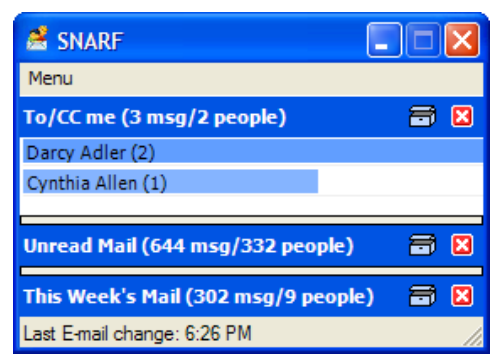

Fig. 2. SNARF collapsed in "Awareness Mode". Clicking any view title will open that view.

electronic communications may not, in the end, account for true social importance. Still, volume seems to be a comprehensible way of approximating interaction.

\section{Concluding Remarks}

SNARF shows one set of experiments into social sorting, as applied to email triage. We believe, however, that these concepts of applying social metadata to end-user tools to be broadly applicable. A number of users seemed particularly interested in using SNARF as a way to get a general overview of their email: who they have interacted with and how often, and to collect general statistics on their informational history. For example, one user commented "[My favorite aspect of SNARF was:] being able to tell who the people are who send me the most mail-and having the evidence to persuade them to send me less!" While much of this social metadata is collected in SNARF database, it is not yet reflected back to users in a general way.

While SNARF may not be for everyone, deploying an application that people could use on their own email on a day-to-day basis allowed us to collect a wealth of data. The deployment illustrated that personally-addressed mail matters, that social metadata can be helpful for staying aware of incoming mail, and that strangers are importantly different from people you know. We also saw challenges in accurately representing people's identities and in handling the lag in social history when people switch communication contexts. These lessons can be applied to future systems that incorporate social metadata.

One of the most popular features of SNARF was the personal views. We are pleased that aspects of this can be directly approximated in other mail clients today. Many email clients support "search folders," dynamic folders that list all email that match given criteria. A search folder of "unread mail, sent to me" allows people to adopt one use of social metadata into their current email experience. Of course there are some drawbacks: the view provided in the search folder would not combine multiple messages by one person, nor would it sort those people by their names.

Finally, we feel one of the strengths of SNARF was the simplicity of the underlying metrics. The notion of "how many emails have I sent to this person" is a comprehensible one and the transparency of the social sorting algorithms allowed users to understand the system. As we begin to take use social metadata in other places, as when auto-completing email addresses or ordering search results, emphasizing simple and straightforward metrics will prevent user confusion 
Acknowledgements. We would like to thank Gina Venolia for valuable survey and user interface design thoughts; in addition, we would very much like to thank our users.

\section{References}

1. Allen, D.: Getting Things Done: The Art of Stress-Free Productivity. Penguin Putnam: New York (2001)

2. Bellotti, V., Ducheneaut, N., Howard, M., Smith, I.: Taking email to task: the design and evaluation of a task management centered email tool. In: Proceedings of CHI, pp. 345-352 (2003)

3. Cutrell, E., Czerwinski, M., Horvitz, E.: Notification, Disruption, and Memory: Effects of Messaging Interruptions on Memory and Performance. In: Proceedings of Interact 2001. Tokyo, Japan (2001)

4. Ducheneaut, N., Bellotti, V.: E-mail as Habitat: An Exploration of Embedded Personal Information Management. Communications of the ACM, pp. 30-38 (2001)

5. Fiore, A., LeeTiernan, S., Smith, M.: Observed Behavior and Perceived Value of Authors in Usenet Newsgroups: Bridging the Gap. In: Proceedings of CHI, pp. 323-330 (2002)

6. Fisher, D., Dourish, P.: Social and temporal structures in everyday collaboration. In: Proceedings of CHI, pp. 551-558 (2004)

7. Gruen, D., Rohall, S., Minassian, S., Kerr, B., Moody, P., Stachel, B., Wattenberg, E., Wilcox, E.: Lessons from the ReMail prototypes. In: Proceedings of CSCW, pp. 152-161 (2004)

8. Horvitz, E., Jacobs, A., Hovel, D.: Attention-Sensitive Alerting. In: Proceedings of UAI '99, pp. 305-313 (1999)

9. Nardi, B., Whittaker, S., Schwartz, H.: NetWORKers and their Activity in Intentional Networks. The Journal of Computer-Supported Cooperative Work 11, 205-242 (2002)

10. Neustaedter, C., Brush, A., Smith, M.: Beyond From and Received: Exploring the Dynamics of Email Triage. In: Proceedings of CHI 2005, pp. 1977-1980 (2005)

11. Neustaedter, C., Brush, A., Smith, M., Fisher, D.: The Social Network and Relationship Finder: Social Sorting for Email Triage. In: Proceedings of the, Conference on Email and Anti-Spam (CEAS) (2005)

12. Takkinen, J., Shahmehri, N.: Café: A Conceptual Model for Managing Information in Electronic Mail. In: Proceedings of the Thirty-First Hawaii International Conference on Software Systems (1999)

13. Tyler, J., Tang, J.C.: When Can I Expect an Email Response? A Study of Rhythms in Email Usage. In: Proceedings of the European Conference on Computer Supported Cooperative Work (ECSCW 2003), ACM Press, New York (2003)

14. Venolia, G.D., Neustaedter, C.: Understanding Sequence and Reply Relationships within Email Conversations: A Mixed-Model Visualization. In: Proceedings of CHI 2003, pp. 361-368 (2003)

15. Venolia, G.D., Dabbish, L., Cadiz, J.J., Gupta, A.: Supporting Email Workflow. Microsoft Technical Report TR-2001-88 (2001) 